\title{
New Journal in Nonlinear Science, Engineering and Mathematics
}

Based on activities of International Symposium on Nonlinear Theory and its Applications in recent two decades, this new journal "Nonlinear Theory and Its Applications" (NOLTA, in short) has started now.

In International Symposium on NOLTA, various researchers have been gathered. Their research fields cover vast topics in nonlinear science, engineering and mathematics. They have interchanged their ideas for a long time. As a result, fruitful co-operations have been born and grew. To make an outstanding journal recording excellent research results including such remarkable co-operative research results is starting idea of designing this journal NOLTA.

First of all, we have decided to make NOLTA as an all-electronic journal published quarterly by the Institute of Electronics, Information and Communication Engineers (IEICE). Then, as the same philosophy of the International Symposium of NOLTA, we have decided that NOLTA covers the entire field of nonlinear science including

1) Nonlinear Theory in Science and Engineering,

2) Nonlinear Phenomena in Science and Engineering,

3) Mathematical and Numerical Methods,

4) Interdisciplinary Fields,

and

5) Applications in Science and Engineering.

Finally, we have designed that the purpose of NOLTA is to publish original peer-reviewed papers on outstanding inventions, innovation, and finding that have influential importance to nonlinear science.

One of the features of NOLTA is that to view papers on NOLTA is free. We believe that free access to papers on NOLTA through Internet as well as article quality make possible to achieve rapid circulations and to give deep impact among researchers in the area of nonlinear science, engineering and mathematics.

Another important feature of NOLTA is to organize in principle special issue or special section in every volume. Purpose of this is to highlight emerging attracting fields in nonlinear science, engineering and mathematics. In this volume, the special issue on Recent Progress in Nonlinear Theory and its Applications is published. The members of the Editorial Committee of NOLTA would like to express their sincere thanks to Professor Tomomasa Nagashima, Guest Editor and other members of Guest Editorial Committee for their enormous efforts for editing the issue.

Members of the Editorial Committee would like to express their sincerely thanks to all peoples who support them in order to start NOLTA. Readers are strongly encouraged to submit their outstanding results to NOLTA.

\section{Shin'ichi Oishi}

Editor-in-Chief 